\title{
Dense optical reference frames: UCAC and URAT
}

\author{
N. Zacharias \\ U. S. Naval Observatory, 3450 Mass. Ave. NW, Washington, DC 20392, USA \\ email: nz@usno.navy.mil
}

\begin{abstract}
A series of ground-based, dedicated astrometric, observational programs have been performed or are in preparation which provide a dense and accurate optical reference frame. Integral to all these programs are new observations to link the Hipparcos Celestial Reference Frame (HCRF) to the International Celestial Reference Frame (ICRF), based on compact, extragalactic radio sources.

The U.S. Naval Observatory CCD Astrograph Catalog (UCAC) 3rd release is in preparation. A pixel re-reduction is in progress to improve astrometric and photometric accuracy as well as completeness of this all-sky reference catalog to 16th magnitude. Optical counterparts of ICRF radio sources have been observed with 0.9 -meter telescopes contemporaneously. Scanning of over 5000 early-epoch astrograph plates on StarScan has been completed. These data will improve the proper motions of stars in the 10 to 14 mag range for the UCAC3 release.

A 111 million-pixel CCD was successfully fabricated in 2006 and test observations at the USNO astrograph are underway. Four of such detectors will be used for the USNO Robotic Astrometric Telescope (URAT) focal plane assembly. Phase I of URAT will use the astrograph to reach 18th magnitude, while the new 0.85 -meter telescope with a 4.5 deg diameter field of view will reach 21 st magnitude. The URAT primary mirror has been fabricated.
\end{abstract}

Keywords. astrometry, reference systems, catalogs, instrumentation: detectors

\section{Introduction}

The primary, optical reference frame currently is the Hipparcos Celestial Reference Frame, HCRF (IAU 2000), which is a subset of the Hipparcos Catalogue (ESA 1997). The HCRF contains about 100,000 selected stars from the brightest to about V = 12 magnitude, with most stars around $\mathrm{V}=8$ to 9. The Tycho-2 Catalogue (Høg, Fabricius, Makarov et al. 2000) contains an almost complete set of the 2.5 million brightest stars to about $\mathrm{V}=11.5$ and is based on both the Hipparcos space mission Tycho instrument data and early-epoch, ground-based catalogs, with the AC2000 (Urban, Corbin, Wycoff et al. 2003) playing a major role. The large epoch difference between these ground-based catalogs and the Hipparcos mission allowed to derive reliable proper motions for Tycho-2 stars at the 1 to 2 mas $\mathrm{yr}^{-1}$ accuracy level.

However, even the Tycho-2 Catalogue can not be utilized as an astrometric standard in most practical applications due to the smallness of the field of view and saturation limiting magnitude of typical sky images taken with today's telescopes. The United States Naval Observatory (USNO) has engaged in programs to further densify the optical reference frame. The USNO CCD Astrograph Catalog (UCAC) project is a completed observing program and is discussed in the next section. The future USNO Robotic Astrometric Telescope (URAT) and its first phase (nicknamed "U-mouse") is presented in Sections 3 and 4, respectively, followed by a brief discussion of merged data products.

There are other dense optical reference star catalogs, which are not discussed here. The USNO-B catalog (Monet, Levine, Canzian et al. 2003) is widely used when deeper data 


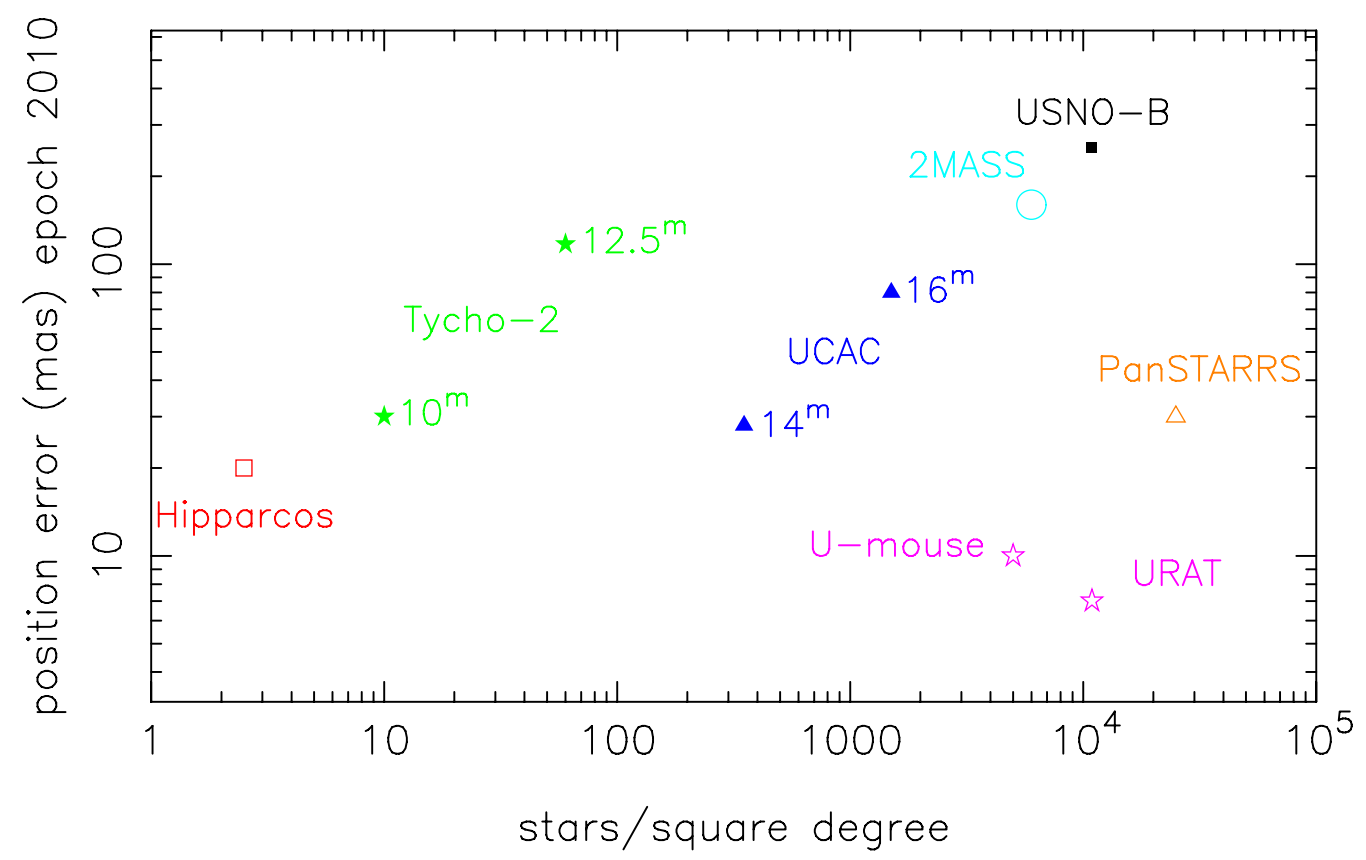

Figure 1. The global positional accuracies of some optical reference star catalogs are plotted as a function of the density (number of stars per square degree). For Tycho-2 and UCAC a range is given for positional errors depending on the magnitude as indicated. See text for further explanation.

than 16th magnitude are needed. The Deep Astrometric Standard (DAS) project (Platais, Wyse, Zacharias 2006) is well underway to reach 25th magnitude in 4 selected areas in the sky (see also these proceedings). The Bordeaux Observatory M2000 (Ducourant, Le Campion, Rapaport et al. 2006) and Carlsberg Meridian Telescope catalogs (Evans, Irwin, Helmer 2002) are examples of other dense optical reference catalogs covering specific declination zones with positional accuracies comparable to those of UCAC. The IAU Working Group on densification of the optical, celestial reference frames is working toward bringing all those catalogs onto a common system.

For comparison, the positional accuracies of current and near future major star catalogs are presented in figure 1. Given are mean standard errors of absolute positions per coordinate, propagated to the epoch of 2010 whenever proper motions are available. The value for PanSTARRS is actually achieved with commissioning data, while the goal is about 10 mas (E. Magnier, private com., see also these proceedings). Values for URAT and U-mouse are conservative estimates based on the UCAC experience.

\section{UCAC}

The UCAC project began in 1996 with the automation of the USNO 8-inch Twin Astrograph. Its visual corrected lens was used for guiding, while a new 5-element "redlens" imaged 1.0 square degree sections of the sky onto a $4 \mathrm{k} \times 4 \mathrm{k}$ CCD. Over 277,000 such images were taken between 1997 and 2004 at the Cerro Tololo Interamerican Observatory (CTIO) and the Naval Observatory Flagstaff Station (NOFS). The first data release in 2000 covers a significant fraction of the southern hemisphere, while the second release, the UCAC2 catalog (Zacharias, Urban, Zacharias, et al. 2004), covers the sky area from the south celestial pole to about $+50^{\circ}$ declination. 
The UCAC2 is widely used because of its density (about 50 million stars) and high astrometric accuracy of about 20 mas for stars in the 10 to 14 mag range. A single bandpass (579 to $642 \mathrm{~nm}$ ) was utilized. All stars in UCAC2 have proper motions which were derived by combining the new epoch CCD data with early-epoch transit circle and photographic data. The UCAC2 was reduced to the International Celestial Reference Frame (ICRF) using Tycho-2 reference stars.

A complete re-processing of the UCAC data is in progress, with a planned final catalog release (UCAC3) in 2008. Major improvements upon the UCAC2 release are: a) complete sky coverage, b) improved completeness, by including for example many double stars, previously skipped "problem" cases and high proper motion stars, c) smaller positional errors at the epoch of observation due to better modeling, d) improved proper motions due to inclusion of new or newly reduced early-epoch data, and e) better photometry due to better modeling and use of a real-aperture photometry.

The test re-processing of UCAC pixel data resulted in the detection of many more faint stars not included in the UCAC2 release. This improved limiting magnitude is obtained by utilizing a more sophisticated dark frame subtraction algorithm than was used in the previous catalog construction. Re-processing of all 4.5 TB compressed raw pixel data is in progress.

Since the release of UCAC2 the StarScan plate measuring program (Zacharias et al. 2008) has been completed. All available (over 1900) plates of the AGK2 project (Schorr, Kohlschütter 1951) as well as the 2300 Hamburg Zone Astrograph and 900 USNO Black Birch program plates were measured. A re-processing of the Northern (Klemola, Jones, Hanson 1987) and Southern (Platais, Girard, Kozhurina-Platais et al. 1998) Proper motion (NPM, SPM) program plates are in progress as well, in a joint effort with Yale University and Lick Observatory.

An integral part of the UCAC program is a direct link to the ICRF radio sources which define the primary celestial reference frame. Images of the ICRF optical counterparts (mostly quasars) were observed mainly with the Kitt Peak National Observatory (KPNO) and CTIO 0.9-meter telescopes with contemporaneous coverage of the same fields by specific astrograph observations, not included in the UCAC2 public release. All relevant extragalactic data are being reduced (Zacharias \& Zacharias 2005) for a check on the optical to radio reference frame link (see also these proceedings).

\section{URAT}

The URAT project will conduct a very accurate (5 mas level for 13 to 18 mag stars) astrometric survey of the entire sky extending to a magnitude limit of 21 , obtaining positions, proper motions and parallaxes based on new observations with a dedicated 0.85 meter aperture and 3.6 meter focal length telescope. The optical design of that telescope with a $4.5^{\circ}$ diameter field of view (Laux, Zacharias 2005) was completed by the EOST company in 2005. The excellent quality primary mirror was delivered to USNO in November 2008. The completion of the telescope optics, tube assembly, and mount depends on future funding.

The development of the URAT focal plane detectors progressed in 2006 by successful fabrication of a single large-format CCD containing 111 million pixels (Bredthauer, Boggs, Bredthauer 2007, Zacharias, Dorland, Bredthauer, et al. 2007). A "10k" camera based on a thinned, back-illuminated version of this STA1600 chip was completed in 2007 by Semiconductor Technology Associates (STA) and saw first light at the USNO astrograph on October 9 (Fig. 2). The URAT camera will consist of 4 of these CCDs plus 4 smaller, rectangular CCDs used for guiding and focus control (Fig. 3). USNO 


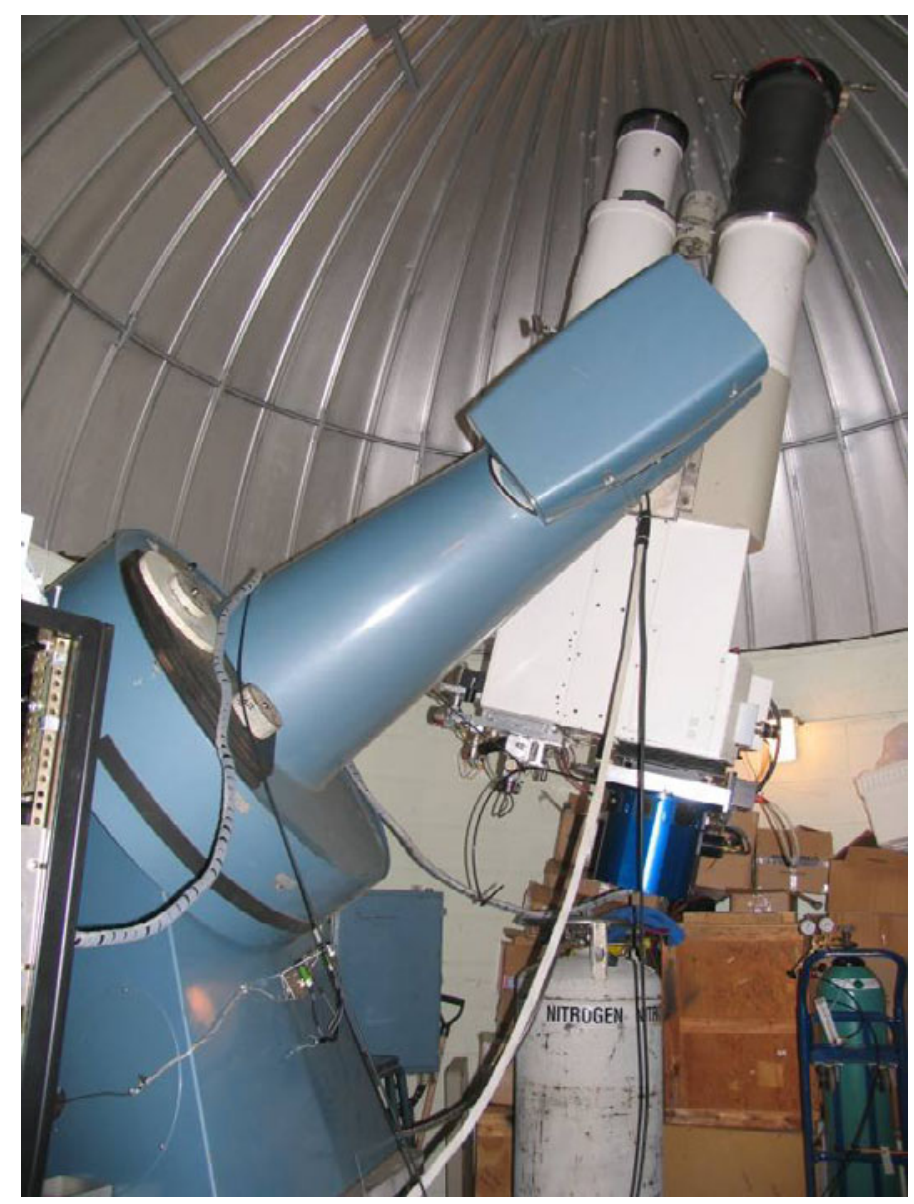

Figure 2. 10k camera with a single STA1600 chip mounted at the USNO astrograph, currently located at the Flagstaff station.

funded this URAT focal plane assembly in 2008. More details about the URAT project and telescope can be found elsewhere (Zacharias 2005).

\section{U-mouse}

With the URAT telescope not complete yet, the URAT focal plane assembly can be put to good use for the "redlens" of the USNO astrograph. The pixel size of the STA1600 chip $(9 \mu \mathrm{m})$ is the same as that of the $4 \mathrm{k}$ camera used for the UCAC project, and the field of view of the "redlens" is $9^{\circ}(324 \mathrm{~mm})$ in diameter, a perfect match for the "4shooter" URAT camera. The resulting project is the first phase of URAT ("U-mouse") and aims at an all-sky survey to 18th magnitude. The gain of 2 magnitudes over the UCAC project using the same telescope comes from a higher quantum efficiency (back vs. front illumination), and the use of longer exposure times (300 sec vs. $125 \mathrm{sec}$ ) in the new survey. Exposure times in the UCAC survey were limited by a high dark current, set to mitigate the CTE effect. Going from 16th magnitude (UCAC) to 18th (U-mouse) gives about a factor of 10 more stars and makes all the difference for projects like PanSTARRS (Magnier 2007) with the anticipated saturation limit near 16th magnitude.

A single exposure of the "4-shooter" camera at the astrograph will cover 27 square degrees of sky and thus will enable several complete overlaps of the accessible sky per 


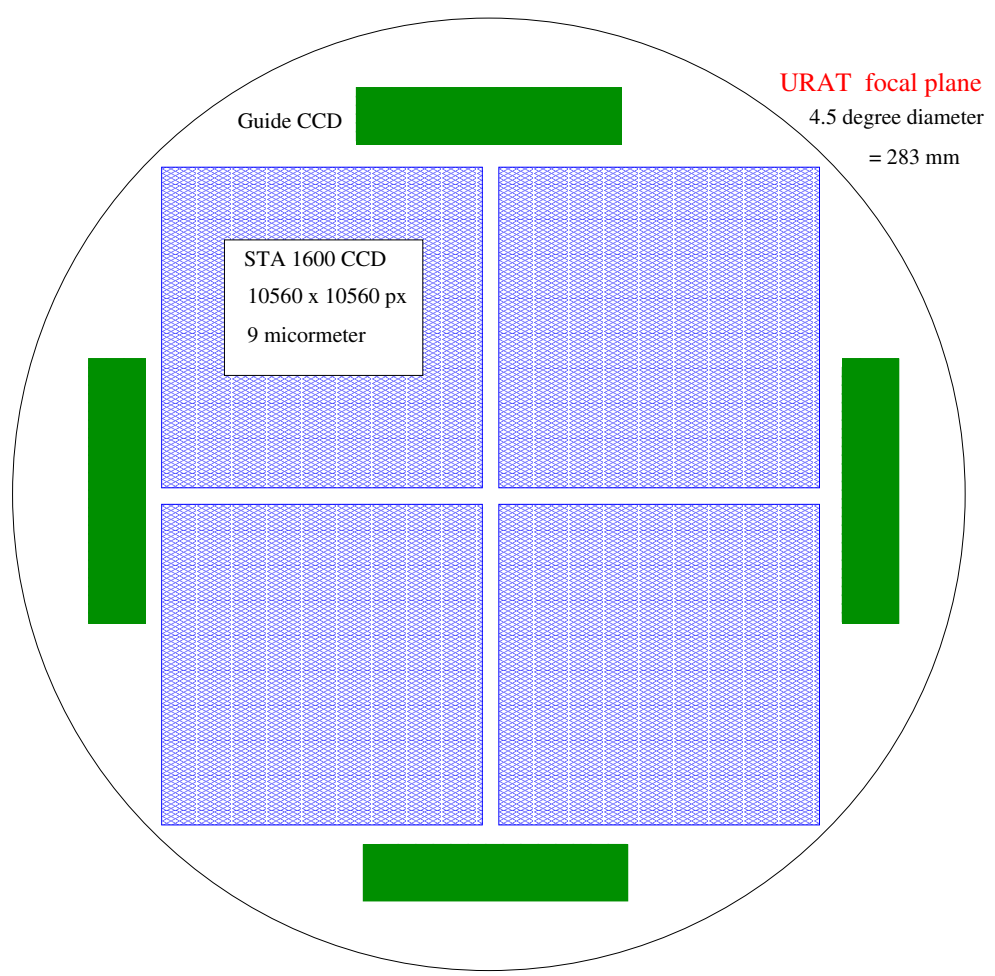

Figure 3. Layout of the 4-shooter focal plane with 4 main CCDs (each 10,560 by 10,560 pixels) and additional, smaller, rectangular CCD chips for guiding and focus control. The same focal plane assembly can be used at both the refurbished USNO astrograph and the future URAT 0.85-meter telescope.

year. Operations are planned to start in 2009 at CTIO after a major re-build of the astrograph (single lens tube assembly) has been completed, which started in 2007.

\section{NOMAD}

The Naval Observatory Merged Astrometric Dataset (www.nofs.navy.mil/nomad) is a first step in combining various star catalogs for dense optical reference frame applications. NOMAD covers all-sky and includes stars from the brightest to the limit of the Schmidt plate surveys. Astrometric information is picked from the Hipparcos, Tycho-2, UCAC, "Yellow-Sky" (NPM, SPM data) and the USNO-B catalogs by a priority algorithm. NOMAD is not a compiled catalog and only a single entry per unique star is picked from the parent catalogs, inheriting also the systematic errors of a particular catalog. The NOMAD astrometric data are supplemented by available optical and 2MASS photometry. Data extracts for small areas in the sky can be obtained online through the above link. The complete NOMAD dataset consists of 90 GB in binary format.

USNO has recently started a long-term project to put all such data and reduction procedures together into a dynamical universal star catalog (S. Levine, private comm). However, it will be a major effort to characterize even the existing star catalogs and remove systematic errors before combining astrometric data. A major step in this direction will be the final UCAC3 release, which is anticipated to be used as a dense optical reference frame for analyzing other star catalogs like the Sloan Digital Sky Survey (SDSS) 
(Pier, Munn, Hindsley et al. 2003) and the Guide Star Catalog (GSC) data (Bucciarelli, Lattanzi, McLean et al. 2008).

\section{Acknowledgements}

Thanks to all members of the UCAC team, the staff at the Cerro Tololo Interamerican Observatory, and the Washington DC and Flagstaff based Naval Observatory personnel involved in these astrometric catalog efforts.

\section{References}

Bredthauer, R., Boggs, K., \& Bredthauer, G. 2007, 2007 Image Sensor Workshop, p.170

Bucciarelli, B., Lattanzi, M. G., McLean, B. et al. 2008, GSC-II catalog release GSC2.3, these proceedings

Ducouraunt, C., Le Campion, J. F., Rapaport, M. et al. 2006, A\&A 448, 1235

European Space Agency 1997, The Hipparcos Catalogue, SP-1200

Evans, D. W., Irwin, M. J., \& Helmer, L. 2002, A\& A 395, 347

Høg, E., Fabricius, C., Makarov, V. V. et al. 2000, A $₫ A$ 355, L27

IAU Resolution B1.2 2000, BAAS 127, 3043, and in proceedings IAU 24th GA, Manchester, p.36

Klemola, A. R., Jones, B. F., \& Hanson, R. B. 1987, AJ 94, 501

Laux, U. \& Zacharias, N. 2005, in Proceedings of "Astrometry in the Age of the Next Generation of Large Telescopes" Eds: Kenneth Seidelmann and Alice K. B. Monet, ASP-CS 338, 106

Magnier, E. 2007, ASP-CS 364, 153

Monet, D. G., Levine, S. E., Canzian, B. et al. 2003, AJ 125, 984

Pier, J. R., Munn, J. A., Hindsley, R. B. et al. 2003, AJ 125, 1559

Platais, I., Girard, T. M., Kozhurina-Platais, V. et al. 1998, AJ 116, 2556

Platais, I., Wyse, R. F. G., \& Zacharias, N. 2006, PASP 118, 107

Schorr, R. \& Kohlschütter, A. 1951, "Zweiter Katalog der Astronomischen Gesellschaft", AGK2, Volume I, Hamburg

Urban, S. E., Corbin, T. E.; Wycoff, G. L. et al. 2003, BAAS 33, 1494 (199th AAS meeting, abstr. 129.04)

Zacharias, N., Urban, S. E., Zacharias M. I., Wycoff G. L., Hall D. M., Monet D. G., \& Rafferty T. J. 2004, AJ 127, 3043

Zacharias, N. 2005, in Proceedings of "Astrometry in the Age of the Next Generation of Large Telescopes" Eds: Kenneth Seidelmann and Alice K. B. Monet, ASP-CS 338, 98

Zacharias M. I. \& Zacharias N. 2005, in P. Kenneth Seidelmann \& Alice K. B. Monet (eds.), Astrometry in the Age of the Next Generation of Large Telescopes, Proc. meeting at Lowell Observatory, Flagstaff, USA (San Francisco: ASP-CS 338) p. 184

Zacharias, N., Dorland, B., Bredthauer, R., Boggs, K., Bredthauer, G., \& Lesser, M. 2007, SPIE 6690 , paper 8

Zacharias, N., Winter, L., Holdenried, E. R., De Cuyper, J.-P., Rafferty, T. J., Wycoff, G. L., 2008 , in press, $P A S P$ 